were: attention problems $(\mathrm{F}=4.935, \mathrm{p}=0.004)$, depression problems $(\mathrm{F}=3.073, \mathrm{p}=0.035)$, $\mathrm{ADHD}(\mathrm{F}=4.422, \mathrm{p}=0.007)$, oppositional defiant $(\mathrm{F}=2.865, \mathrm{p}=0.045)$, and total $\mathrm{t}$-score $(\mathrm{F}=3.073$, $\mathrm{p}=0.035$ ). Maternal mean DBI scores were also statistically significantly different when grouped by days of maternal dysfunction due to sleepiness $(\mathrm{F}=9.791, \mathrm{p}<0.001)$. There was no relation between these CBCL categories and maternal DBI scores. DISCUSSION/ SIGNIFICANCE OF IMPACT: Maternal self-reported days of dysfunction due to sleepiness may potentially increase risk for their children to develop further psychopathology independent of mothers' depression symptomatology. These findings highlight the need for broader assessment clinically of children's environments with additional focus on maternal function given the potential impact on their children's functional outcomes.

Nonclinical factors associated with contralateral prophylactic mastectomy among breast cancer patients in the Surveillance, Epidemiology, and End Results (SEER) database

Timothy Paul Copeland ${ }^{1}$, Jill Dworsky, Kesav Raghavan and Benjamin Franc

${ }^{1}$ David Geffen School of Medicine at UCLA

OBJECTIVES/SPECIFIC AIMS: The study aims to measure the associations between nonclinical factors and the likelihood of electing contralateral prophylactic mastectomy [(CPM) i.e. bilateral mastectomy for unilateral cancer] among women with breast cancer, with a focus on the roles of race, relationship status, and geographic location. The outcome of interest is a dichotomized surgery type variable (i.e. CPM versus other surgery). METHODS/STUDY POPULATION:. The Surveillance, Epidemiology, and End Results registry was queried to identify female breast cancer patients diagnosed at stage IA through IIIC from 2010 through 2015 and received surgery as part of their primary treatment $(n=174,776)$. A multilevel logistic regression was used to model likelihood of CPM versus less aggressive surgical treatment (i.e. breast conserving surgery or unilateral mastectomy). Fixed-effects included age at diagnosis, race, relationship status, insurance type, county-level median income, county-level population density, stage at diagnosis (low-stage, IA-IIB; advanced-stage, IIIA-IIIC), an interaction term between race and stage at diagnosis, and breast tumor subtype. County of residence was used as a random-effect. RESULTS/ANTICIPATED RESULTS: Among women with low-stage cancer, compared to the reference class of white women, black women had 0.57 times lower odds of CPM $(\mathrm{p}<0.0001)$, Hispanic women had 0.69 times lower odds of CPM $(\mathrm{p}<0.0001)$, and Asian women had 0.60 times lower odds of CPM $(\mathrm{p}<0.0001)$. Among women with advancedstage cancer, compared to white women, black women had 0.42 times lower odds of CPM $(\mathrm{p}<0.0001)$, Hispanic women had 0.51 times lower odds of CPM $(\mathrm{p}<0.0001)$, and Asian women had 0.45 times lower odds of CPM $(\mathrm{p}<0.0001)$. Compared to the reference class of single, never-married women, divorced/separated women had 1.25 times greater odds of CPM $(\mathrm{p}<0.0001)$, widowed women had 1.11 times greater odds of CPM ( $\mathrm{p}=0.009)$, and married/partnered women had 1.18 times greater odds of CPM $(\mathrm{p}<0.0001)$. County-level variation from the random-effect (MOR, 1.49; $\mathrm{p}<0.001$ ) had a greater influence on CPM election than fixed-effects for insurance class, breast tumor subtype, county median income, county population density, and year of surgery. DISCUSSION/SIGNIFICANCE OF IMPACT: The nonclinical factors associated with variation in breast cancer surgical decisionmaking suggest patients and providers both may benefit from further education about surgical treatment options. Providers may also benefit from educational materials that highlight treatment selection disparities within specific contexts, such as surgery for primary unilateral breast cancer. To more narrowly tailor future policy interventions, an additional mixed-methods exploration is recommended to clarify how relationship status and location serve as mechanisms for breast cancer decision-making.

3451

\section{Pain, Quality of Life, and Emotional Measures as Predictors for Outcomes following Surgery for Nerve Injuries}

Kartemus Heary ${ }^{1}$, Madeline Thompson ${ }^{1}$, Alex Wong ${ }^{1}$, Jana Dengler ${ }^{1}$, Victoria Kaskutas ${ }^{1}$, Benjamin Philips ${ }^{1}$ and Susan Mackinnon ${ }^{1}$

${ }^{1}$ Washington University in St. Louis

OBJECTIVES/SPECIFIC AIMS: Examine data from PNID patients to evaluation the strength of associations between pre-operative and post-operative levels of pain, quality of life, and emotional reactions to pain to determine if one or more can serve as better predictors of surgical success than pain. METHODS/STUDY POPULATION: In our preliminary study, we gathered data from a pre-existing database of 464 PNID patients that contains self-reported visual analog scale scores (VAS) of pain intensity, QoL, and depression. We measured these variables at three time points: pre-operatively, post-operatively, and at the final visit. We used the Wilcoxon signed rank test to determine if each of these three variables differed significantly between the pre-operative visit and the post-operative visit period and from the pre-operative visit to the final visit. RESULTS/ANTICIPATED RESULTS: Median time from the pre-operative visit to surgery was 9 weeks; median time from surgery to the post-operative visit was 4 weeks; and median time from the post-operative visit to the final visit was 23.5 weeks. There was a clinically meaningful difference in pain scores between the pre-operative and post-operative visits (median difference 1.15 ; 95\% CI $0.75-1.55$ ). In the period between the post-operative visit and the final visit there was also a decrease in pain (0.90; 95\% CI 0.55-1.30). The magnitude of change in median difference of 1.85 (95\% CI 1.50-2.20) between the pre-operative visit and the final visit was larger than the change in median difference of 0.90 (95\% CI 0.55-1.30) between the post-operative visit and the final visit. The pre-operative visit median QoL score was higher than the median score at the post-operative visit (1.65; 95\% CI 1.25-2.10). The smallest median difference in QoL of occurred between the post-operative and the final visit (1.10; 95\% CI 0.60-1.45). As seen with the pain scores, the magnitude of change in median difference of 2.50 (95\% CI 2.20-2.85) for QoL was greatest between the preoperative and the final visit. Depression scores showed the least amount of change amongst all the variables, between the preoperative and the post-operative visit (1.00; 95\% CI (0.70-1.40), and similarly between the post-operative visit and the final visit $(0.15 ; 95 \%$ CI (0-.40). The median differences between the pre-operative and final visit were greatest in QoL (2.50; 95\% CI 2.20-2.85), followed by pain scores $(1.85$; $95 \%$ CI $1.50-2.20)$, and finally, depression (1.05; 95\% CI 0.70-1.40). DISCUSSION/SIGNIFICANCE OF IMPACT: Our results show that all three variables measured improve with surgery and continue to improve over the postoperative course to the final visit. This suggest that the relationships between pain, QoL, and depression should be further investigated. We are hopeful that elucidating how these variables interact in the 